Bond University

Research Repository

\title{
Physiological Profile of Male Competitive and Recreational Surfers
}

Furness, James; Hing, Wayne; Sheppard, Jeremy M; Newcomer, Sean; Schram, Ben; Climstein, Mike

Published in:

Journal of Strength and Conditioning Research

DOI:

10.1519/JSC.0000000000001623

Licence:

Unspecified

Link to output in Bond University research repository.

Recommended citation(APA):

Furness, J., Hing, W., Sheppard, J. M., Newcomer, S., Schram, B., \& Climstein, M. (2018). Physiological Profile of Male Competitive and Recreational Surfers. Journal of Strength and Conditioning Research, 32(2), 372-378. https://doi.org/10.1519/JSC.0000000000001623

\section{General rights}

Copyright and moral rights for the publications made accessible in the public portal are retained by the authors and/or other copyright owners and it is a condition of accessing publications that users recognise and abide by the legal requirements associated with these rights.

For more information, or if you believe that this document breaches copyright, please contact the Bond University research repository coordinator. 
1 MANUSCRIPT TITLE: THE PHYSIOLOGICAL PROFILE OF MALE

2 COMPETITIVE AND RECREATIONAL SURFERS

RUNNING HEAD: PHYSIOLOGICAL PROFILE OF SURFING

5

6

7

8

9 
ABSTRACT

Surfing consists of both high and low intensity paddling of varying durations, utilizing both the aerobic and anaerobic systems. Surf specific physiological studies lack adequate group sample sizes and $\mathrm{VO}_{2 \text { peak }}$ values are yet to determine differences between competitive and recreational surfers. The purpose of this study was therefore to provide a comprehensive physiological profile of both recreational and competitive surfers. This multi-site study involved 62 male surfers, recreational $(n=47)$ and competitive $(n=15)$. Anthropometric measurements were conducted followed by DEXA, anaerobic testing and finally aerobic testing. $\mathrm{VO}_{2 \text { peak }}$ was significantly greater in competitive compared to recreational surfers ( $M$ $=40.71 \pm 3.28$ vs. $31.25 \pm 6.31 \mathrm{ml} / \mathrm{kg} / \mathrm{min}, p<.001)$. This was also paralleled for anaerobic power $(M=303.93$ vs. $264.58 \mathrm{~W})$ for competitive surfers. Arm span and lean total muscle mass was significantly $(p \leq .01)$ correlated with key performance variables $\left(\mathrm{VO}_{2 p e a k}\right.$ and anaerobic power). No significant $(p \geq .05)$ correlations were revealed between season rank and each of the variables of interest ( $\mathrm{VO}_{2 \text { peak }}$ and anaerobic power). Key performance 
The basic physiological requirements of surfing has remained unchanged for over a 1,000 years in that a surfer paddles a board out to the waves, then rides it back to shore (22). Through the use of time motion analysis, the sport can be further broken down into periods of repetitive upper body movement during paddling and prolonged periods of sitting, interspersed with intermittent explosive lower body and trunk movements (20). Several studies have revealed that paddling is the predominant aspect of surfing and encompasses approximately $50 \%$ of a surfing session or competitive heat $(9,19,26,30)$. The activity requirements of a 20 minute heat in young competitive surfers using global positioning system (GPS) technology has previously been analyzed. Results revealed that $54 \%$ of the total time involved paddling with a mean heart rate of $140 \pm 11.6$ beats/min (9). The majority of these paddling bouts (60\%) were only 1 to 20 seconds long; highlighting the importance of short intense paddling. The activity requirements for young recreational surfers revealed similar results with paddling encompassing 42.6 to $44 \%$ of the total time and mean heart rates ranging between $128 \pm 13$ to $135 \pm 6.9$ beats/min $(19,26)$.

It is apparent that both forms of surfing are intermittent in nature, and clearly utilize the aerobic and anaerobic energy systems. It could be suggested that surfers must possess a highly developed capacity to physiologically recover in short rest periods before recommencing high intensity paddling bouts. Aerobic $\left(\mathrm{VO}_{2 p e a k}\right)$ and anaerobic (peak watts) physiological testing through paddling assessment have previously been assessed in several studies $(8,15,16,19,21)$.

Loveless and Minahan (15) conducted the only study which compared competitive and recreational surfers and revealed no significant differences between the groups for $\mathrm{VO}_{2 \text { peak }}$ 
values. Mendez-Villanueva et al. (21) also revealed no difference in $\mathrm{VO}_{2 \text { peak }}$ scores when European level surfers were compared against regional level surfers. Only two studies $(8,15)$ have assessed peak power output using ergometers; discrepancies in mean peak power out values are evident between studies. Competitive surfers have been shown to possess significantly $(p<.05)$ greater peak power outputs $(8,15)$ and season rank has been significantly $(p<.05)$ correlated with peak power output (8).

A key theme in these physiological studies is the variation in $\operatorname{VO}_{2 \text { peak }}$ values $(M=37.8$ to $54.2 \mathrm{ml} / \mathrm{kg} / \mathrm{min})$ and peak power outputs $(M=205$ to $348 \mathrm{~W})$. An explanation for the variations may be due to differences in equipment and testing protocols used. In addition, there appears to be no difference in $\mathrm{VO}_{2 \text { peak }}$ scores between recreational and competitive surfers, despite this being a common finding in most other sports. It should be noted that all of these studies investigating $\mathrm{VO}_{2 \text { peak }}$ lack adequate group sample sizes $(n<10)$. This limits the ability to reveal meaningful mean differences between groups and generalize results to surfing cohorts.

In conjunction with physiological assessment, several studies have also assessed body composition in both recreational and competitive surfers. Surfers have generally been considered to possess moderate levels of body fat ranging from 10.5 to $22 \%(10,17,20)$. Only one study has revealed significant differences between body composition between surfing cohorts (29). The interpretation of these results is limited given that body composition was assessed through skinfolds. It has been shown that varying the skinfold site by as little as $1 \mathrm{~cm}$ produces significantly different results when experienced practitioners measure the same subject (1). Dual energy x-ray absorptiometry (DEXA) has been shown to be extremely reliable in estimating body composition (6) and has yet to be used in a surfing population. 
It is apparent that further physiological testing is needed in a larger sample size comparing recreational and competitive surfers. Therefore, the aims of this study were; 1 ) to provide the aerobic and anaerobic profile for competitive and recreational surfers and determine if differences exist between groups; 2) to provide the body composition and anthropometric comparisons for competitive and recreational surfing cohorts and; 3) to determine if physiological testing could be used in a surf specific screen to assist with discriminating in performance. It is hypothesized that competitive cohorts will have increased anaerobic and aerobic power and decreased body fat compared with recreational surfers.

\section{METHODS}

\section{Experimental Approach to the Problem}

Physiological variables ( $\mathrm{VO}_{2 \text { peak }}$ and anaerobic power), anthropometrics and body composition measurements were determined at multiple study sites on both competitive and recreational surfers. A comparative analysis was conducted between key performance variables $\left(\mathrm{VO}_{2 \text { peak, }}\right.$ relative anaerobic power and peak anaerobic power) of both competitive and recreational groups to determine significant differences.

\section{Subjects}

This was a multi-site study that involved a total of 62 male surfers, recreational $(n=47$; age $26.50 \pm 5.28$ years; mass $77.42 \pm 10.69 \mathrm{~kg}$; height $180.13 \pm 7.54 \mathrm{~cm})$ and competitive $(n=$ 15; age $26.73 \pm 4.68$ years; mass $77.83 \pm 6.62 \mathrm{~kg}$; height $179.44 \pm 3.96 \mathrm{~cm})$. The 15 competitive surfers were competing on the World Qualifying Series (WQS) or world championship tour (WCT) (surfing experience $18.86 \pm 5.46$ years; surfing frequency $13.23 \pm$ 4.54 hours per week; dry land training $4.5 \pm 2.35$ hours per week) all remaining surfers were 
classified as recreational (surfing experience $13.22 \pm 6.93$ years; surfing frequency $7.56 \pm$ 4.91 hours per week; dry land training $2.57 \pm 2.93$ hours per week). To be classified as a recreational surfer, subjects were to have at least one year experience, currently be surfing and not compete higher than local club level. A total of 34 (54.8\%) were tested at one Australian University and the remaining 28 were tested at an American University; where only aerobic testing was conducted. Subjects were tested following their normal routine of sleep, nutrition and hydration levels prior to testing. Being a multi-site study ethics was granted through the University Human Research Ethics Committee (RO1610) and through the Institutional Review Board for the Protection of Human Subjects (IRB, 2013-118) prior to commencement. Participants were informed of the risks and benefits of the investigation prior to signing an informed consent form.

Prior to undertaking analysis between the competitive and recreational groups, data collected between both testing sites needed to be analyzed to ensure there were no differences in $\mathrm{VO}_{2 \text { peak, }}$ mass and age. Only aerobic testing was conducted at the American University and therefore only key variables that could influence $\mathrm{VO}_{2 \text { peak }}$ scores were analyzed. No significant differences were seen between the two sites for age (27.19 \pm 4.24 vs. $26.03 \pm 5.91$ years; $p$ $=.47)$, weight $(\mathrm{M}=74.82 \pm 8.66$ vs. $79.20 \pm 11.70 \mathrm{~kg} ; p=.17)$, and $\mathrm{VO}_{2 \text { peak }}(32.75 \pm 5.24$ vs. $30.25 \pm 6.85 \mathrm{ml} / \mathrm{kg} / \mathrm{min} ; p=.19)$. Therefore data was pooled together to provide a recreational group of 47 surfers.

\section{Procedures}

Testing at the Australian University was conducted by a physiotherapist with additional training in exercise testing and an accredited exercise physiologist with over 20 years' 
experience. Testing at the American University was conducted under the direct supervision of an exercise physiologist with over 15 years of experience. Initially, anthropometric measurements were conducted followed by DEXA then anaerobic testing and finally aerobic testing. All subjects were tested in a University setting and underwent the exact same order of testing on the same day; however testing conducted at the American University involved aerobic testing only.

\section{Anthropometrics and Body composition}

Anthropometric measurements included height, mass and arm span. Height was initially measured to the nearest $0.1 \mathrm{~cm}$ and body mass was measured with minimal clothing using a standard medical balance scale (Seca, 700, Hamburg, Germany). Arm span was measured to the nearest $0.1 \mathrm{~cm}$ according to standard recommendations (23). Arm span was divided by height to determine “Ape Index”; a ratio commonly used with sports such as rock climbing and swimming where larger ratios favour the competing athlete (31).

A DEXA scanner (General Electric, Prodigy Pro (Madison, Wisconsin, USA)) was utilized for all body composition testing. Encore software provided an output of segmental body composition for each surfer (right \& left arms, legs and trunk). All scans were completed according to the standardized DEXA operational protocol (24). Surfers were centrally positioned where by both feet were placed on a foam block and foam pads were placed on each hand to help determine tissue differences between arms and trunk (foam is transparent under DEXA). Using a foam block and pads, a constant distance between feet $(15 \mathrm{~cm})$ and between hands and trunk $(3 \mathrm{~cm})$ was maintained. According to standardized baseline conditions (24) subjects are required to be overnight fasted on the morning of measurements. Unfortunately the DEXA occurred prior to anaerobic and aerobic testing and therefore 
overnight fasting was not appropriate. To ensure standardized conditions, subjects were required to fast for at least 2 hours prior to testing.

\section{Anaerobic power output testing}

Both aerobic and anaerobic testing was completed on a wind-braked swim bench ergometer (Vasa, Inc., Essex Junction, VT, USA) with the addition of a surfboard mounted on top of the bench. A new display unit with interoperability (ANT+) technology was used to gather all data on the display unit of the swim bench ergometer. This allowed for total peak power, left and right peak power, total distance covered and velocity to be calculated and captured. Total peak power was defined as the highest sample of left plus right watts (W).

The resistance unit on the swim bench ergometer provided seven airflow resistance settings. The highest setting was used in this study, as previous research by Loveless and Minahan (16) revealed the maximum power output was achieved at the highest resistance. Anaerobic power output was measured during a 10-second sprint on the swim bench ergometer at maximal effort (completed prior to aerobic testing). The surfer was initially familiarized with the equipment and given standardized instructions on the testing procedures. This was followed by a three-minute warm up at 30 watts and then three 5-second maximal effort sprints with each sprint separated by a 20-second rest period. Following the completion of the warm up the surfer had a 10-minute rest before completing the 10-second sprint at maximal effort. A 10 minute rest period was selected as complete resynthesis of adenosine triphosphate (ATP) occurs within three to five minutes, and complete creatine phosphate resynthesis can occur within eight minutes $(4,11,13)$. This protocol was based on previous anaerobic testing conducted on a competitive surfing cohort $(8,16)$. As previously discussed the inclusion of ANT+ software allows for data on the display unit to be capture and 
wirelessly transmitted. Peak power, mean power, left and right power outputs, peak velocity and total distance were all calculated.

\section{Aerobic $\mathrm{VO}_{2 p e a k}$ uptake testing}

Subjects’ $\mathrm{VO}_{2 \text { peak }}$ was obtained during an incremental endurance exercise test. Measuring peak oxygen consumption is considered the gold standard for quantifying aerobic fitness.

Swim bench ergometry has previously been shown to be both valid and reliable to test peak aerobic and anaerobic levels in recreational and competitive surfers $(8,15)$. All surfers underwent aerobic testing on the swim bench ergometer. Oxygen consumption was analyzed using a Parvo Medics (TrueOne $\left.{ }^{\circledR}, 2400\right)$ automated gas analysis system $\left(\mathrm{O}_{2}\right.$ analyser, $\mathrm{CO}_{2}$ analyser, pneumotach) which was calibrated prior to each test. The expired gas analysis system meets Australian Institute of Sport accreditation standards for precision and accuracy. This provided breath-by-breath measurement of maximum oxygen consumption (L/min), and relative to body weight ( $\mathrm{ml} / \mathrm{kg} / \mathrm{min}$ ), maximal ventilation, and energy expenditure (kcals). Oxygen uptake was averaged every 30 seconds, with the peak value recorded as the highest value obtained over a 30-second period.

The incremental test began at 30 watts, with increments of 10 watts every minute. Testing was terminated if maximum heart rate was exceeded, respiratory exchange ratio (RER) reached greater than 1.5, oxygen consumption did not increase concurrently with power output, required power output was not maintained for greater than 10 seconds, volitional exhaustion was achieved or any symptoms of chest pain were expressed by the surfer. This termination criteria was based upon the ACSM guidelines for exercise testing and prescription (3). The incremental testing protocol was based off previous $\mathrm{VO}_{\text {2peak }}$ testing 

the surfboard attached to the swim bench is seen in Figure 1.

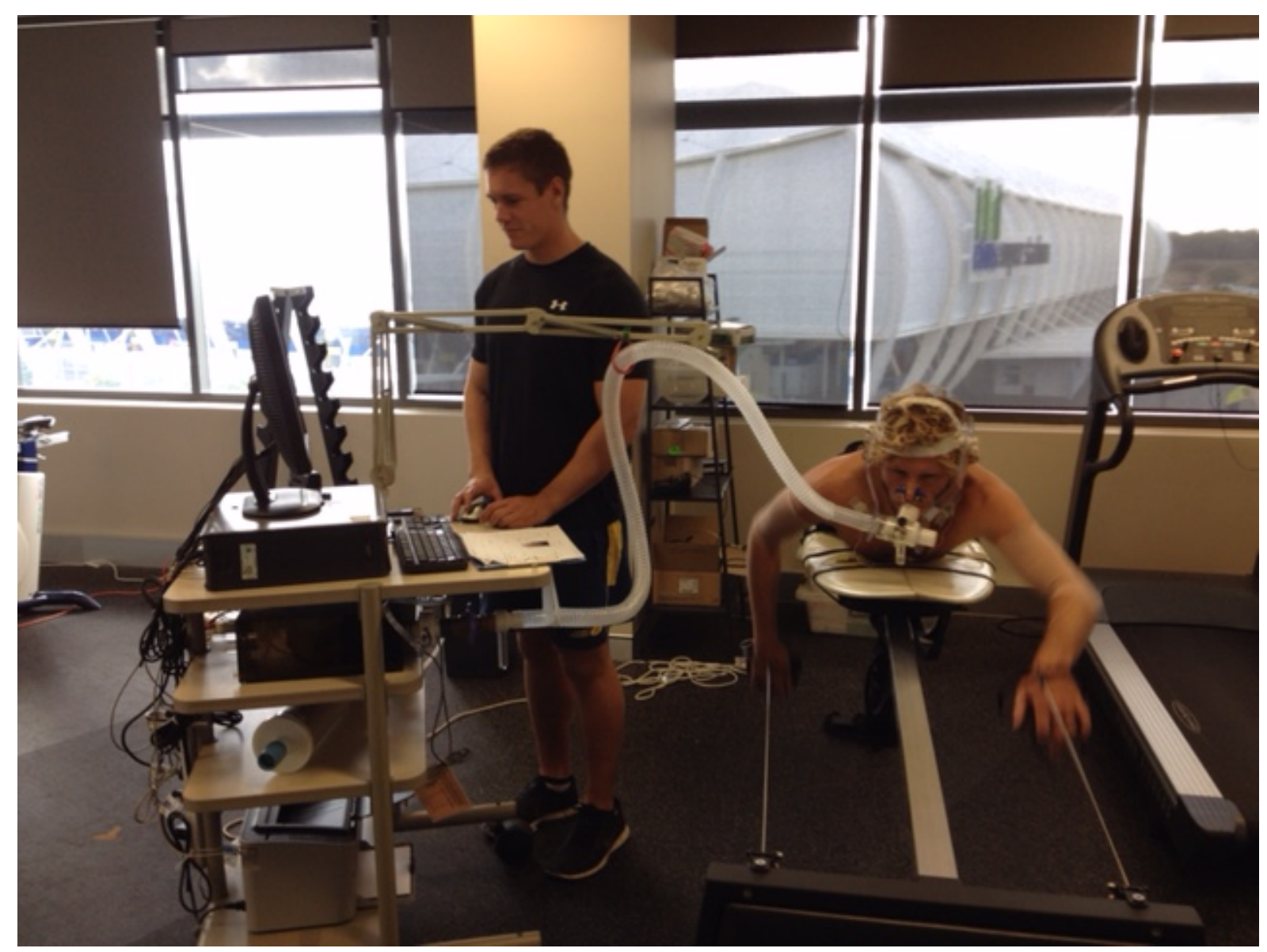

Figure 1: Laboratory setup of $\mathrm{VO}_{2 p e a k}$ testing performed on the swim bench ergometer 


\section{Statistical Analyses}

Data analysis was performed with SPSS version 20.0. Descriptive statistics including means, standard deviations and ranges were calculated for each measure and for each session. A Shapiro-Wilks test ( $>$ >0.05) (27) and a visual inspection of their frequency histograms, normal Q-Q plots and box plots showed that all key performance variables $\left(\mathrm{VO}_{2 p e a k}\right.$, relative anaerobic power and peak anaerobic power) were normally distributed for both the competitive and recreational groups; with the magnitude of skewness and kurtosis being nonsignificant $(5,7)$. Independent $t$-tests were used for comparative analysis between competitive and recreational groups. Paired $t$-tests were used to determine differences within groups. A Spearman's rank order correlation was conducted between end of year ranking and

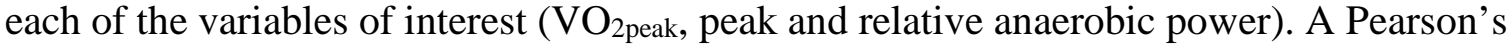
correlational analysis was conducted with key physical attributes (arm span and total muscle mass) and key performance variables ( $\mathrm{VO}_{2 \text { peak, }}$ peak anaerobic power and relative anaerobic power). 


\section{RESULTS}

\section{Reliability analysis}

A small pilot study was conducted for both anaerobic $(n=7)$ and DEXA $(n=8)$ assessment. Whereby, each subject was assessed twice on the same day separated by 2 hours. The same assessor completed each assessment in order to evaluate intra-rater reliability. ICC scores were within the excellent range for anaerobic testing and the use of DEXA (ICC .97 and .99 respectively). Reliability of $\mathrm{VO}_{2 \text { peak }}$ testing has been well established with test retest scores being high $(r=.95-.99)(2)$.

\section{Recreational vs. Competitive}

A comparative analysis between the competitive and recreational groups can be seen in Table 1. Independent $t$-tests revealed significant differences $(p \leq .05)$ between recreational and competitive groups for key performance variables. Competitive surfers had significantly greater arm span ( $M=190.61$ vs. $182.61 \mathrm{~cm}, p=.01)$ compared to recreational surfers. Consequently competitive surfers revealed significantly higher Ape Index scores (arm span/ height) compared to recreational males ( $M=1.06$ vs. $1.03, p<.001) . \mathrm{VO}_{2 p e a k}$ was and peak anaerobic power was significantly greater in the competitive surfers compared to recreational surfers ( $M=40.71$ vs. $31.25 \mathrm{ml} / \mathrm{kg} / \mathrm{min}, p<.001 ; M=303.93$ vs. $264.58 \mathrm{~W}$ respectively).

\section{Physical attributes and key performance variables}

Arm span was significantly $(p \leq .01)$ correlated with $\mathrm{VO}_{2 \text { peak }}(r=.55)$, relative anaerobic power $(r=.49)$ and peak power output $(r=.72)$. Total muscle mass was also significantly correlated $(p \leq .05)$ with $\mathrm{VO}_{2 \text { peak }}(r=.56)$, relative anaerobic power $(r=.49)$ and peak power output $(r=.83)$. 
249 A total of 10 competitive male surfers were utilized in the analysis as all of these surfers

250

251

252

253

254

255

256

257

258

259

260

261 completed an entire year of competition. Key variables of interest were $\mathrm{VO}_{2 \text { peak, }}$ peak anaerobic power and relative anaerobic power. No significant correlations $(p \geq .05)$ were revealed for each of the variables of interest ( $\mathrm{V}_{2 \text { peak }}, r=.33$; peak anaerobic power, $r=.06$; relative anaerobic power, $r=.09$ ).

\section{Symmetry in power outputs}

As power output data was attained during both the anaerobic and aerobic testing, comparisons between dominant and non-dominant arm outputs were conducted using paired $t$-tests. There was no statistical difference $(p>.05)$ between mean dominant and nondominant arm power outputs for anaerobic (dominant $=139.14 \pm 34.30$ versus non-dominant $=135.62 \pm 2.59 \mathrm{~W}$ ) and aerobic testing (dominant $=31.40 \pm 5.77$ versus non-dominant $=$ $31.05 \pm 5.53 \mathrm{~W}$ ) for all surfers. 
Table 1: Key physical attributes and performance variables for competitive and recreational surfers (M \pm SD)

\begin{tabular}{|c|c|c|c|}
\hline Measure & Competitive; $n=15$ & Recreational; $n=47$ & $p$ value \\
\hline \multicolumn{4}{|c|}{ Anthropometrics and Body Composition } \\
\hline Arm span (cm) $\dagger$ & $190.61 \pm 4.79$ & $182.61 \pm 9.28$ & $.01^{*}$ \\
\hline Ape Index $\dagger$ & $1.06 \pm 0.01$ & $1.03 \pm 0.02$ & $<.001^{*}$ \\
\hline Total body fat (\%) $\dagger$ & $17.11 \pm 2.93$ & $18.86 \pm 3.33$ & .12 \\
\hline Total muscle mass (g) $\dagger$ & $61.66 \pm 4.02$ & $58.21 \pm 6.46$ & .81 \\
\hline \multicolumn{4}{|l|}{ Aerobic $\mathrm{VO}_{2 p e a k}$ test } \\
\hline $\mathrm{VO}_{2 \text { peak }}(\mathrm{L} / \mathrm{min})$ & $3.14 \pm 0.37$ & $2.41 \pm 0.53$ & $<.001^{*}$ \\
\hline $\mathrm{VO}_{\text {2peak }}(\mathrm{ml} / \mathrm{kg} / \mathrm{min})$ & $40.71 \pm 3.28$ & $31.25 \pm 6.31$ & $<.001^{*}$ \\
\hline $\begin{array}{c}\text { Respiratory exchange ratio } \\
\text { (RER) }\end{array}$ & $1.10 \pm 0.07$ & $1.21 \pm 0.08$ & $<.001^{*}$ \\
\hline Peak blood lactate (mmol) & $12.01 \pm 3.28$ & $12.03 \pm 3.37$ & .99 \\
\hline Peak heart rate (b.min $\left.{ }^{-1}\right)$ & $182.07 \pm 5.27$ & $175.58 \pm 10.51$ & $.03^{*}$ \\
\hline $\begin{array}{l}\text { Age predicted heart rate max } \\
\qquad(\%)\end{array}$ & $94.41 \pm 4.19$ & $90.80 \pm 5.53$ & $.03 *$ \\
\hline Peak aerobic power (W) & $121.93 \pm 9.20$ & $101.26 \pm 18.49$ & $<.001^{*}$ \\
\hline \multicolumn{4}{|l|}{ Anaerobic 10s test } \\
\hline $\begin{array}{c}\text { Absolute peak anaerobic } \\
\text { power }(W) \dagger\end{array}$ & $303.93 \pm 57.99$ & $264.58 \pm 46.14$ & $.04 *$ \\
\hline Mean anaerobic power $(\mathrm{W}) \dagger$ & $257.21 \pm 47.28$ & $224.04 \pm 39.75$ & $.03^{*}$ \\
\hline $\begin{array}{c}\text { Relative anaerobic power } \\
\qquad(\mathrm{W} / \mathbf{k g}) \dagger\end{array}$ & $3.91 \pm 0.63$ & $3.53 \pm 0.38$ & $.04 *$ \\
\hline Peak anaerobic speed $(\mathrm{m} / \mathrm{s}) \dagger$ & $1.65 \pm 0.09$ & $1.54 \pm 0.10$ & $<.001^{*}$ \\
\hline
\end{tabular}

$\dagger$ refers to testing conducted at Bond University only $(\mathrm{n}=34)$; * refers to statistical significance $(p \leq 0.05)$ determined through independent $t$-tests; NA refers to "not applicable". 


\section{DISCUSSION}

The purpose of this study was to 1) to provide the aerobic and anaerobic profile of competitive and recreational surfers and determine if differences exist between groups; 2) to provide the body composition and anthropometric comparisons for competitive and recreational surfing cohorts and 3) to determine if physiological testing could be used in a surf specific screen to assist in discriminating performance. Findings from the current study support our hypothesis that competitive surfers tested on a swim bench ergometer had significantly higher values for both oxygen consumption and anaerobic power. In contrast to our hypothesis body composition measured by DEXA did not significantly differ between competitive and recreational surfers tested in this study.

\section{Aerobic Testing}

Time motion analysis revealed that upper body paddling represents the largest component of surfing (20). The competitive group had significantly higher aerobic scores in comparison to the recreational group. These findings suggest that high levels of aerobic fitness are attributes associated with competitive surfers. This is logical when considering the activity requirements of a competitive heat and the associated additional training. Farley et al. (9) reported that during a 20 minute competitive heat a surfer is required to participate in repeated high and low intensity paddling bouts (1 to 20 seconds) interspersed with short rest periods accumulating $54 \pm 6.3 \%$ of the total heat time . This paddling requirement may foster a high capacity for oxygen uptake in order to allow for sufficient recovery between paddling bouts. High intensity interval training has previously been shown to increase maximal oxygen consumption (12). Given that paddling is characterized by a series of short sprints it may be these demands of competitive surfing that cause increases in maximal oxygen consumption. Competitive surfers are also generally involved in additional training that is designed to 
replicate paddling bouts in heats. This is commonly achieved using interval type training methods (25).

The findings from the current study have both similarities and inconsistencies with previous surf specific research $(8,15,16,19,21)$. The competitive $\mathrm{VO}_{2 \text { peak }}$ scores are similar to previous research conducted by Farley, Harris and Kilding (8) and Loveless and Minahan (15); however the recreational scores appear to be consistently lower than previous research conducted by Loveless and Minahan (15) and Meir, Lowdon and Davie (19). All of the aforementioned studies had sample sizes of less than 10, thus limiting the ability to compare their results with the current study and generalize their results to recreational and competitive surfing cohorts. The current study revealed significant differences in $\mathrm{VO}_{2 \text { peak }}$ scores between recreational and competitive surfers. Previous research $(15,21)$ had not identified this, however both of these studies had sample sizes of less than 10 surfers in each group; once again limiting the ability to generalize the results to a surfing population.

\section{Anaerobic Testing}

As previously mentioned $60 \%$ of paddling bouts were 1 to 20 seconds long, highlighting the importance of short intense paddling (9). This activity requirement utilizes the anaerobic energy system and hence the need to attempt to replicate this activity on a swim bench. This study revealed significantly higher anaerobic scores in competitive surfers compared to recreational surfers (see Table 1). This is an important attribute to a competitive surfer as it assists in the ability to catch waves and gain a position advantage over their competitors during a heat. It may also allow for fast entry into a wave optimizing the execution of manoeuvres (28). It needs to be highlighted that competitive surfers commonly take part in additional training to further develop this energy system; therefore higher anaerobic scores in the competitive group may be due to both the activity requirements of surfing in heats and 
additional training. Nevertheless, this information adds to the physiological profile of a competitive and recreational surfer.

Only two published studies have conducted anaerobic testing in a surfing cohort using upper limb ergometers $(8,16)$. Our results are slightly higher than the study conducted by Farley, Harris and Kilding (8); however a kayak ergometer was used which differs to the swim bench ergometer used in the current study. Loveless and Minahan (16), using the same equipment set-up, revealed slightly higher values for the competitive surfers (348 $\pm 78 \mathrm{~W}$ ) compared with the results of the current study (303.93 $\pm 57.99 \mathrm{~W}$ ). This inconsistency remains puzzling considering that the average weight for the study by Loveless and Minahan (16) was $61.1 \pm$ $9.2 \mathrm{~kg}$ compared to the current study's average weight of $77.83 \pm 6.62 \mathrm{~kg}$. The current study revealed a significant correlation $(r=.83 ; p<.001)$ between lean muscle mass and peak power output; therefore it would be expected that the heavier competitive group would produce greater peak power output scores. It needs to be noted that Loveless and Minahan (16) conducted six trials over two days to determine the mean power output of $348 \pm 78$. It could be postulated that a learning effect occurred with subjects becoming more proficient at the motor pattern required and the demands of the test over the six trials.

\section{Body Composition}

This study was the first to utilize DEXA to determine body composition with the variable of interest being percent body fat. Results revealed competitive surfers have low to moderate levels of body fat (17\%). This is not surprising as surfers are not purely endurance athletes who tend to reveal lower body fat levels ranging from 8-13\% through the use of DEXA (24). The results of the current study are similar to previous research, which has used skinfold assessment to estimate body fat with values ranging from $10.5-22 \%$ for competitive male and female surfers $(10,17,20)$. It could be postulated that low body fat values do not represent a 
real advantage from a performance perspective. It has also been suggested that higher body fat levels are possibly an adaptation to surfing in colder waters as additional body fat provides greater insulation $(18,20)$. Once again, this information adds to building the profile for recreational and competitive surfers using DEXA.

\section{Performance Screening}

The final aim of this study was to determine if physiological testing could be used to discriminate in performance. Significant differences were revealed between competitive and recreational surfers indicating the ability of the aerobic and anaerobic testing to discriminate between groups. However, when analysing the competitive cohort separately, no associations were detected. Whereby a surfers ranking and key performance variables (peak and relative power and $\mathrm{VO}_{2 \text { peak }}$ ) were not correlated. This finding suggested that although high anaerobic and aerobic levels are associated with competitive surfers they do not assist in determining their individual level of performance. This is logical as a surfer is ranked according to their ability of actually riding a wave (performing critical manoeuvres) which was not assessed with these physiological tests. Therefore, although paddling assessment is crucial to undertake, it does not assist in discriminating the level of performance within a competitive cohort. It should however be noted that the standard deviations for key performance variables (VO2 $2_{\text {peak, }}$ peak and relative power output) were all minimal indicating most results were closely related. Perhaps a test which resulted in a wide spread data set may have illustrated a stronger correlation. However, a single study conducted by Farley, Harris and Kilding (8) has previously shown a correlation between season rank and anaerobic scores achieved during a 10-second paddle sprint. 
Interestingly a correlational analysis revealed significant $(p \leq .05)$ associations between arm span, lean muscle mass and key performance variables $\left(\mathrm{VO}_{2 \text { peak, }}\right.$, peak and relative power output). These results may suggest that those surfers with longer arms and greater lean muscle mass produced higher $\mathrm{VO}_{2 p e a k}$ and anaerobic scores. Correlations between arm span and $\mathrm{VO}_{2 \text { peak }}$ scores are commonly reported in swimming studies $(14,23)$. There were no differences in height between the competitive and recreational group; however, arm span significantly differed as with the ratio of arm span divided by height, known as "Ape Index". This finding is unique as it raises the question as to whether significant increases in arm span in the competitive group are a result of a physical predisposition for success in the sport. Further investigation of this variable is warranted to determine the utility of this indices for assisting in talent identification.

Finally, this is the first surf specific study to analyse symmetry of power output during aerobic and anaerobic paddling tests. No statistical difference was found between the dominant and non-dominant arms for power outputs during either test. This finding is novel in itself as it provides information that symmetry of power output is needed during paddling. This opens up several practical applications; where-by surfers suffering shoulder injuries could use swim bench ergometers for corrective and feedback purposes. It could also be used as a screening tool to identify asymmetry or even for rehabilitative purposes.

To our knowledge, this study is the largest comparative surf specific study to date that has comprehensively presented the physiological profile of competitive and recreational surfers. Key performance variables ( $\mathrm{VO}_{2 \text { peak, }}$ peak and relative power output) are significantly higher in competitive surfers indicating this is both an adaptation and requirement in this cohort. Interestingly no significant correlation was identified between key performance variables and ranking in the competitive cohort. This suggests tests which replicate wave-riding 
components, may be more appropriate to discriminate performance within a competitive group. Arm span and ape index were the anthropometric measurements that were significantly greater in the competitive group; whether this is a result of physical predisposition is yet to be determined. This comprehensive study adds to the physiological and physical profile of a recreational and competitive surfer. This battery of physiological tests could be used as a screening tool to identify an athlete's weaknesses or strengths. Coaches and clinicians could then select appropriate training regimes to address weaknesses and therefore place less emphasis on strengths.

There is also potential for this research within the surfing industry. Prior to the arrangement of sponsoring deals, a surfer could undergo physiological screening to provide the company with additional information. This concept is not foreign to many other sports and may be of benefit to both the athlete and the company providing the sponsorship. Whereby, the surfer is provided with a profile of his or her strengths and weakness along with strategies to address their weaknesses. The company is provided with additional information regarding the state of the athlete from a physiological point of view.

\section{PRACTICAL APPLICATIONS}

Key performance variables $\left(\mathrm{VO}_{2 \text { peak }}\right.$ and anaerobic power) are significantly higher in competitive surfers indicating this is both an adaptation and requirement in this cohort. This battery of physiological tests could be used as a screening tool to identify an athlete's weaknesses or strengths. Coaches and clinicians could then select appropriate training regimes to address weaknesses. These findings are limited to the current study and results 
411 should not be generalized to female surfing cohorts as further research is needed in this 412 surfing cohort. 


\section{REFERENCES}

1. Ackland TR, Lohman TG, Sundgot-Borgen J, Maughan RJ, Meyer NL, Stewart AD, and Müller W. Current status of body composition assessment in sport. Sports Med 42: 227-249, 2012.

2. Adams G and Beam W. Exercise physiology laboratory manual Boston: McGraw Hill, 2008.

3. Armstrong LE, Brubaker PH, Whaley MH, and Otto RM. ACSM's Guidelines for Exercise Testing and Prescription. Baltimore: Lippincott Williams \& Wilkins, 2006.

4. Baechle TR and Earle RW. Essentials of strength training and conditioning. Champaign, IL: Human Kinetics, 2008.

5. Barnes J. Fundamental statistics for social research: Step-by-step calculations and computer techniques using SPSS for windows. J Child Psychol Psychiatry 39: 1055-1055, 1998.

6. Carver T, Christou N, and Andersen R. In vivo precision of the GE iDXA for the assessment of tota body composition and fat distribution in severely obese patients. Obesity 21: 1367-1369, 2013.

7. Cramer D. Fundamental Statistics for Social Research: Step-by-Step Calculations and Computer Techniques Using SPSS for Windows. Hoboken: Taylor and Francis, 2012.

8. Farley O, Harris NK, and Kilding AE. Anaerobic and aerobic fitness profiling of competitive surfers. J Strength Cond Res 26: 2243-2248, 2012.

9. Farley O, Harris NK, and Kilding AE. Physiological demands of competitive surfing. J Strength Cond Res 26: 1887-1896, 2012.

10. Felder JM, Burke LM, Lowdon BJ, Cameron-Smith D, and Collier GR. Nutritional practices of elite female surfers during training and competition. Int J Sport Nutr 8: 36, 1998.

11. Harris RC, Edwards RHT, Hultman E, Nordesjö LO, Nylind B, and Sahlin K. The time course of phosphorylcreatine resynthesis during recovery of the quadriceps muscle in man. Pflugers Arch 367: 137-142, 1976.

12. Helgerud J, Hoydal K, Wang E, Karlsen T, Berg P, Bjerkaas M, Simonsen T, Helgesen C, Hjorth N, Bach $\mathrm{R}$, and Hoff J. Aerobic high-intensity intervals improve VO2max more than moderate training. Med Sci Sports Exerc 39: 665-671, 2007.

13. Hultman E and Sjoholm H. Biochemical causes of fatigue. In: Human Muscle Power. NL Jones, N McCartney, AJ McComas, eds. Champaign, IL: Human Kinetics, 1986, pp 215-235.

14. Jurimae J, Haljaste K, Cicchella A, Latt E, Purge $P$, Leppik A, and Jurimae T. Analysis of swimming performance from physical, physiological, and biomechanical parameters in young swimmers. Pediatr Exerc Sci 19: 70-81, 2007.

15. Loveless DJ and Minahan C. Peak aerobic power and paddling efficiency in recreational and competitive junior male surfers. Eur J Sport Sci 10: 407-415, 2010a.

16. Loveless DJ and Minahan C. Two reliable protocols for assessing maximal-paddling performance in surfboard riders. J Sports Sci 28: 797-803, 2010b.

17. Lowdon BJ. The somatotype of international surfboard riders. Sports Med 12: 34-39, 1980.

18. Lowdon BJ and Pateman NA. Physiological parameters of international surfers. Sports Med 12: 30-33, 1980. 
19. Meir RA, Lowdon BJ, and Davie AJ. Heart rates and estimated energy expenditure during recreational surfing. Aust J Sci Med Sport 23: 70-74, 1991.

20. Mendez-Villanueva A and Bishop D. Physiological aspects of surfboard riding performance. Sports Med 35: 55-55, 2005.

21. Méndez-Villanueva A, Perez-Landaluce J, Bishop D, Fernandez-García B, Ortolano R, Leibar X, and Terrados N. Upper body aerobic fitness comparison between two groups of competitive surfboard riders. J Sci Med Sport 8: 43-51, 2005.

22. Moser P. Pacific Passages: An Anthology of Surf Writing. Honolulu: University of Hawaii Press, 2008.

23. Moura $T$, Costa M, Oliveira S, Júnior MB, Ritti-Dias R, and Santos M. Height and body composition determine arm propulsive force in youth swimmers independent of a maturation stage. J Hum Kinet 42: 277-284, 2014.

24. Nana A, Slater GJ, Hopkins WG, Halson SL, Martin DT, West NP, and Burke LM. Importance of standardized DXA protocol for assessing physique changes in athletes. Int J Sport Nutr Exerc Metab 45: 178-185, 2014.

25. Secomb JL. Review of the physical and physiological demands of surfing and suggested training modalities and exercises. JASC 20: 22-33, 2012.

26. Secomb JL, Sheppard JM, and Dascombe BJ. Time-motion analysis of a 2-hour surfing training session. Int J Sports Physiol Perform 10: 17-22, 2015.

27. Shapiro SS and Wilk MB. An analysis of variance test for normality. Biometrika 52: 591, 1965.

28. Sheppard JM, McNamara P, Osborne M, Andrews M, Borges OT, and Chapman DW. Association between anthropometry and upper-body strength qualities with sprint paddling performance in competitive wave surfers. J Strength Cond Res 26: 3345-3348, 2012.

29. Sheppard JM, Nimphius S, Haff G, Tran T, Spiteri T, Brooks H, Slater G, and Newton RU. Development of a comprehensive performance-testing protocol for competitive surfers. Int J Sports Physiol Perform 8: 490-495, 2013.

30. Watsford M, Murphy A, and Coutts A. Energy expenditure and time-motion analysis during recreational surfing. J Sci Med Sport 9: 9, 2006.

31. Watts PB, Joubert LM, Lish AK, Mast JD, and Wilkins B. Anthropometry of young competitive sport rock climbers. Br J Sports Med 37: 420-424, 2003. 